\title{
El estudio de las identidades desde un enfoque cualitativo. La multi-metodología autobiográfica extendida y los talleres lúdico-reflexivos
}

\author{
Qualitative methods in the study of identities. Multi-method \\ autobiographical extended approach and reflexive-ludic \\ workshops
}

\author{
María del C. Peña-Cuanda \\ Universidad Intercultural de Chiapas \\ mariacuanda@yahoo.com.mx (MÉXICO) \\ Moises Esteban-Guitart \\ Universidad de Girona \\ moises.esteban@udg.edu (ESPAÑA)
}

Recibido: 26.102011

Aceptado: 22.05.2013

\section{RESUMEN}

Este artículo revisa dos estrategias de investigación cualitativa (la multi-metodología autobiográfica extendida y los talleres lúdico-reflexivos) que combinan técnicas grupales, visuales y participativas como el dibujo identitario, el juego de roles, el círculo significativo o el cronograma. Se ilustran las distintas técnicas a partir de ejemplos extraídos de estudios que tienen el objetivo de estudiar las identidades. Argumentamos que la aplicación de estos métodos en el contexto de la investigación cualitativa permite captar las interpretaciones que realizan los participantes de las distintas cuestiones con sus propias palabras, significados y asociaciones. Concluimos con la necesidad de devolver a los participantes la interpretación que se realiza del corpus empírico, puesto que son considerados co-investigadores dado su papel activo y de afección recíproca en el proceso del trabajo de campo en este tipo de estudios.

EMPIRIA. Revista de Metodología de Ciencias Sociales. N. ${ }^{\circ}$ 26, julio-diciembre, 2013, pp. 175-200. ISSN: 1139-5737, DOI: 10.5944/empiria.26.7157 


\title{
PALABRAS CLAVE
}

Metodología cualitativa, identidad, metodología autobiográfica, talleres lúdico-reflexivos, métodos visuales.

\begin{abstract}
This article reviews two qualitative methods (the multi-method autobiographical extended approach and the reflexive-ludic workshops) based on visual and participative research techniques such as the self-portrait, role playing, significant circle or timeline. Examples of these techniques are drawn from their application in studies aims to explore identities. The article argues that applying these drawing and participative methods in the context of a qualitative research can up participants' interpretations of questions, and allow a creative way of interviewing that is respondive to participants' own meanings and associations. The article discusses the importance to inform to participants about the interpretation of empirical corpus. Participants are considered coresearchers due to their active role in the process of collecting data in this kind of qualitative methods.
\end{abstract}

\section{KEY WORDS}

Qualitative methodology, Identity, autobiographical methodology, reflexiveludic workshops, visual methods.

\section{INTRODUCCIÓN}

El principal propósito de este artículo es describir, e ilustrar, dos estrategias cualitativas (la multi-metodología autobiográfica extendida y los talleres lúdicoreflexivos) que tienen el objetivo de estudiar las identidades de los participantes en el estudio. Para ello dividimos el artículo en cuatro apartados. En primer lugar, definimos, con la ayuda de Giddens, el concepto de identidad y notamos dos posturas, o modos de entenderla, a saber: la postura esencialista y la postura nominalista. En segundo lugar, describimos las dos estrategias metodológicas propuestas, así como algunas técnicas de investigación asociadas a ellas que tienen el propósito de suscitar «crónicas del yo», es decir, relatos alrededor de uno/a mismo/a. Finalmente, a modo de conclusión, destacamos los beneficios de las estrategias propuestas sin obviar sus dificultades: la necesidad de disponer de marcos teóricos sólidos y específicos para poder realizar un buen análisis del material empírico.

EMPIRIA. Revista de Metodología de Ciencias Sociales. N. ${ }^{\circ}$ 26, julio-diciembre, 2013, pp. 175-200. ISSN: 1139-5737, DOI: 10.5944/empiria.26.7157 


\section{La naturaleza reflexiva y biográfica de la identidad. la perspectiva de giddens}

Las cuestiones en torno a la identidad están presentes en ciencias sociales desde prácticamente su nacimiento. Buen ejemplo de ello son las obra de Cooley (1902) y Mead (1934), en sociología, o las de Erikson (1950) y Tajfel (1984), en psicología. Sin embargo, actualmente cobran especial protagonismo debido a la incertidumbre, fragilidad e inestabilidad que caracteriza lo que Giddens (1997) llama «modernidad tardía», Beck (1998) «sociedad del riesgo» o Bauman (2000) «modernidad líquida».

A pesar de los distintos matices y diferencias, se podría argumentar que dichos autores enfatizan la caducidad de la sociedad industrial debido a que los riesgos e incertidumbres existentes a nivel social, político, económico e individual tienden cada vez más a escapar a las instituciones de control y protección de las sociedades industriales tradicionales. Según lo resume el mismo Bauman (2003: 58):

«En resumen: se ha acabado la mayoría de los puntos de referencia constantes y solidamente establecidos que sugerían un entorno social más duradero, más seguro y más digno de confianza que el tiempo que duraba una vida individual. Se ha acabado la certeza de que "volveremos a vernos», de que estaremos viéndonos repetidamente y durante un largo tiempo futuro, y de que, por tanto, puede suponerse que la sociedad tiene una larga memoria y que lo que hoy nos hagamos mutuamente nos confortará o atormentará en el futuro; de que lo que nos hagamos recíprocamente tendrá una importancia algo más que episódica.»

La angustia por perder el trabajo, por una posible fallida de la empresa, por el envejecimiento de los conocimientos, por la crisis de pareja, la incertidumbre alrededor del terrorismo global, la contaminación o el sistema económico -en definitiva la crisis de los mecanismos tradicionales de confianza- deriva en un aumento de lo que Giddens (1997) llama «reflexividad social», es decir, un aumento en la reflexión (auto-confrontación) sobre las circunstancias en las que desarrollamos nuestras vidas. Precisamente el mismo autor entiende que la identidad es un producto biográfico resultado de la reflexión alrededor de proyectos de vida que se abren y discuten al peligrar las certezas y seguridades de antaño. Según sus propias palabras: «la identidad del yo se constituye por medio del ordenamiento reflejo de la crónica del yo» (Giddens, 1997: 294). En realidad, la identidad se convierte en una tarea en el contexto de las posibilidades de elección. Es un producto narrativo que se crea, mantiene, revisa y formula a través de las actividades (acciones) reflejas del individuo: «es el yo entendido reflexivamente (reflejamente) por la persona en función de su biográfica» (Giddens, 1997: 72).

Decimos que es un «producto narrativo reflejo» ya que para Giddens (1997) la identidad es el resultado de la «crónica del yo», es decir, el «relato o relatos por los que tanto el individuo en cuestión como los demás entienden reflejamente la identidad del yo» (Giddens, 1997: 293). «¿Qué hacer? ¿Cómo actuar? 
¿Quién ser? Son cuestiones fundamentales para cualquier que viva en las circunstancias de la modernidad tardía, y a las que respondemos todos en uno y otro plano, discursivamente o por medio de nuestro comportamiento social diario» (Giddens, 1997: 93).

\section{Dos posturas teóricas en el estudio de las identidades}

En realidad, hoy en día podemos encontrar tantas definiciones de identidad como trabajos sobre ella se han desarrollado. A pesar de esta situación, entre los diversos autores podemos encontrar una conciencia generalizada o consenso en relación a dos puntos: por un lado, la polisemia y dispersión de la noción de identidad (Dubar 2002; Hall, 1996; Giménez 2000; Valenzuela, 2000) y, por el otro, el posicionamiento dualista que ha marcado profundamente las corrientes de su conceptualización (Alcoff, 1989; Hall 1999; Riquer 1992).

Por un lado, está la postura «esencialista», que básicamente remite a la conceptualización de la identidad como mismidad, aquello que nos da la cualidad de idéntico (de ahí el nombre), como sustancia inmutable o categoría natural compartida y transmitida, es el eidos (esencia) de las personas o grupos, por lo tanto algo que es permanente y atemporal. Esta postura está profundamente enraizada en el pensamiento filosófico y en el origen etimológico de la palabra.

La otra postura, más reciente y a la que podríamos situar el enfoque de Giddens anteriormente expuesto, es definida como «nominalismo» (Dubar 2002), postura «constructivista» (Hall 1996, 1999; Giménez 2000; Castells 1999) o «posicional» (Alcoff 1989). La idea básica es que la identidad está sometida a cambios y transformaciones, remite a los modos en que nos identificamos y a las acciones específicas que realizamos y a través de las cuales nos experimentamos mediante actos de identificación. Según esta postura, las identidades se construyen a partir de las formas de nombrar o nombres con que caracterizamos nuestras pertenencias, desde las posiciones enunciadas que se asumen en la representación de un «yo» o un «nosotros» y remiten a una noción limítrofe, contradictoria, ambivalente o de frontera entre lo social-cultural y lo individual o subjetivo.

Por lo que hace a la posición esencialista, a pesar de que algunos autores (Maalouf, 1999) consideran que se ha superado conceptualmente, se reconoce su eficacia discursiva y simbólica en muchos de los movimientos sociales que han emergido o cobrado fuerza en la época actual y que se han sustentado en la identidad como fundamento de movilización y cohesión social, partiendo de algún o algunos atributos que presuponen idénticos, permanentes o ahistóricos, aunque las movilizaciones de estos grupos están fundamentadas en procesos históricos de colonizaciones, exclusiones, discriminaciones, marginaciones, maltratos, negaciones, resistencias, confrontaciones, etc. (Maalouf, 1999) No obstante, esta situación no derriba la postura nominalista o constructivista, pues la identidad retomada como bandera construida desde esencialismos en los movimientos 
sociales puede ser pensada como una posición asumida para la acción política (Alcoff, 1989) o como reunificación imaginaria que posiciona a los sujetos como actores políticos (Hall, 1999).

En Chiapas, un caso emblemático del uso y apropiación de la postura esencialista en los procesos de construcción de representaciones que producen actores políticos situados fue y sigue siendo el movimiento armado del zapatismo, actualmente convertido en un movimiento ideológico. En este caso la reunificación imaginaria cobró sentido desde la identidad de ser indígena, borrando la diversidad lingüística, cultural, religiosa e ideológica que existe entre las distintas comunidades indígenas.

Justamente las investigaciones que hemos desarrollado y en las que hemos empleado las metodologías, que exponemos a continuación, se sitúan en la segunda perspectiva teórica, pues se pregunta por los puntos críticos de diferencia, por las construcciones subjetivas de los participantes intentando propiciar lo que con Giddens llamábamos anteriormente «crónicas del yo».

\section{DOS ESTRATEGIAS METODOLÓGICAS PARA EL ESTUDIO DE LAS IDENTIDADES EN CIENCIAS SOCIALES}

La «multi-metodología autobiográfica extendida» —en adelante MMAEy los «talleres lúdico-reflexivos» —en adelante TLR - son estrategias participativas que permiten estudiar la identidad bajo una perspectiva cualitativa. Eso conlleva asumir dos cosas: en primer lugar, como se ha sugerido anteriormente, se parte de la premisa según la cual la identidad humana es un producto narrativo vinculado a las formas de relatarse, ya sean lingüísticas o visuales (Esteban-Guitart, 2012a; Peña-Cuanda, 2010). En segundo lugar, un enfoque cualitativo conlleva interesarse por la subjetividad, vivencia o experiencia de los participantes, entendiendo que la subjetividad está vehiculada a procesos históricos, políticos, sociales y culturales (González Rey, 2007; Peña-Cuanda, 2008; Ratner, 2012). Lo que ambos métodos pretenden es ofrecer contextos dinámicos (con actividades particulares y tareas asociadas) a través de los cuales los participantes puedan reflexionar y explicitar los sentidos y significados asociados a sí mismos. A continuación explicamos su naturaleza y procedimiento para, posteriormente, ilustrar algunas técnicas asociadas a su aplicación y desarrollo.

\section{La multi-metodología autobiográfica extendida (MMAE)}

La «multi-metodología autobiográfica» fue originariamente una estrategia desarrollada por Bagnoli (2004) con el objetivo de estudiar la identidad de jóvenes inmigrantes en Europa. La propuesta consiste en la combinación de técnicas lingüísticas (entrevista, diario personal) y visuales (utilización de 
fotografías, el dibujo identitario) con la finalidad de facilitar la generación de narrativas identitarias por parte de los participantes en el estudio que se convierten, en términos de Bagnoli (2004: 1), en «co-investigadores». Este método asume el carácter narrativo y dialógico de la identidad, al considerar que ésta consiste en una historia de vida en la que toman cita eventos pasados, presentes, así como futuros imaginados que uno o una cuenta utilizando voces culturales de personas significativas, ya sea positiva o negativamente. Más que las asunciones teóricas alrededor de la identidad y del modelo de Bagnoli, lo que aquí queremos resaltar son los aspectos metodológicos. Simplemente decir que según la autora, la combinación de técnicas cualitativas es la mejor manera de captar las identidades, entendidas como construcciones dialógicas y narrativas.

El objetivo de esta estrategia metodológica consiste en fomentar la reflexividad de los participantes alrededor de sí mismos. Lo que interesa es documentar la identidad de las personas desde su perspectiva. En este sentido, documentar las propias explicaciones y narraciones para, de este modo, comprender los sueños, aspiraciones y visiones que los participantes tienen en relación a sí mismos.

Como ya se ha dicho, originariamente el método consistía en la utilización y combinación de cuatro técnicas o herramientas que explicaremos más adelante y que ahora simplemente anunciamos, a saber: el dibujo identitario, el diario personal, la utilización de fotografías y una entrevista - modelo historia de vida- que se realiza antes y después del proceso metodológico. Un proceso que suele extenderse a un mínimo de una semana. En la primera sesión, el investigador realiza la entrevista con el participante y le explica las otras tres técnicas que el entrevistado deberá realizar en los próximos siete días (hacer un diario personal durante una semana, hacer un dibujo sobre sí mismo o sí misma y, finalmente, recoger distintas fotografías). Finalmente, se realiza otra entrevista con el objetivo de discutir y compartir el material que el participante ha elaborado y recogido. El resultado final es un retrato holístico de la subjetividad del participante en sus propios términos o palabras. Posteriormente, como hemos dicho, detallaremos las tareas - técnicas - ahora solamente mencionadas.

A partir de esta propuesta se desarrolla la «multi-metodología autobiográfica extendida» (MMAE) con el objetivo de revisar las cuatro técnicas del procedimiento original para complementarlas con otros recursos, herramientas o técnicas cualitativas (Esteban-Guitart, 2012b). En concreto, la MMAE consiste en un total de 15 técnicas que se ponen a disposición del investigador, que elige unas u otras en función de su pregunta de investigación y su estudio particular. Las técnicas se agrupan en cuatro categorías que aparecen en la tabla 1, a saber: 1) entrevistas en profundidad, 2) la tarea del dibujo identitario revisada, 3) la detección de artefactos, rutinas y formas de vida y, finalmente, 4) la creación de mapas psicológicos o psicogeomapas.

EMPIRIA. Revista de Metodología de Ciencias Sociales. N. ${ }^{\circ}$ 26, julio-diciembre, 2013, pp. 175-200. ISSN: 1139-5737, DOI: 10.5944/empiria.26.7157 
Tabla 1. La multi-metodología autobiográfica extendida (MMAEA)

\begin{tabular}{|c|c|c|c|}
\hline $\begin{array}{c}\text { Entrevistas } \\
\text { en profundidad }\end{array}$ & $\begin{array}{l}\text { La tarea del dibujo } \\
\text { identitario revisada }\end{array}$ & $\begin{array}{c}\text { Detección de } \\
\text { artefactos, rutinas } \\
\text { y formas de vida }\end{array}$ & $\begin{array}{c}\text { Mapas psicológicos } \\
\text { o psicogeomapas }\end{array}$ \\
\hline $\begin{array}{l}\text { 1. Historia de vida } \\
\text { (modelo de McA- } \\
\text { dams) } \\
\text { 2. Entrevista «Fon- } \\
\text { dos de Conoci- } \\
\text { miento» (proyecto } \\
\text { de Arizona) } \\
\text { 3. Entrevista basada } \\
\text { en Durand (2011) }\end{array}$ & $\begin{array}{l}\text { 4. Dibujo identitario } \\
\text { 5. Definiciones } \\
\text { identitarias } \\
\text { 6. La tarea de las } \\
\text { diez definiciones }\end{array}$ & $\begin{array}{l}\text { 7. Diario personal } \\
\text { de una semana } \\
\text { 8. Detección de ar- } \\
\text { tefactos } \\
\text { 9. Rutinas a través } \\
\text { de fotografías } \\
\text { 10. Rutinas educa- } \\
\text { tivas a través de } \\
\text { fotografías }\end{array}$ & $\begin{array}{l}\text { 11. Cronograma } \\
\text { 12. Genograma } \\
\text { 13. Ecomapa } \\
\text { 14. Geomapa } \\
\text { 15. Mapa relacional } \\
\text { o «círculo signi- } \\
\text { ficativo» }\end{array}$ \\
\hline
\end{tabular}

La misma Bagnoli (2009) propone otras técnicas para complementar la información que se puede obtener al aplicar la «multi-metodología autobiográfica» tradicional, a saber: un tipo de «mapa relacional» y el «cronograma». Además de estas dos, se sugiere la necesidad de incorporar otras herramientas con el fin de ofrecer un amplio abanico al investigador en la realización de una investigación alrededor de la identidad, en particular, o la subjetividad, en general.

Además, una diferencia clave entre la MMA y la MMAE es que la última incorpora la psicología cultural (Cole, 1996; Esteban-Guitart, 2010) como modelo explicativo, introduciendo conceptos como el de «fondos de conocimiento»o el de «artefacto».

Por «fondos de conocimiento» se quiere decir «cuerpos de conocimientos culturalmente desarrollados e históricamente acumulados y a las destrezas esenciales para el funcionamiento y bienestar familiar o individual» (Moll, 1997: 47). Se trata de recursos, habilidades, relaciones, conocimientos que todas las familias, al tener experiencias vitales (por ejemplo laborales) acumulan. En este sentido, la socialización de las personas ocurre en medio y a través de estos particulares fondos de conocimiento que en muchas ocasiones son desconocidos, incluso infravalorados o devaluados, por la cultura escolar. El programa educativo «fondos de conocimiento» consiste en que los maestros detectan los fondos de conocimiento de las familias de sus alumnos con el objetivo de conectarlos con la práctica escolar (Esteban-Guitart y Vila, 2013; González, Moll y Amanti, 2005).

Finalmente, por «artefactos» queremos decir recursos o productos culturales, a la vez físicos y simbólicos, modificados a lo largo de la historia de la humanidad, que tienen el propósito de dirigir la conducta humana (Cole, 1996). Un despertador, por ejemplo, permite levantarse por la mañana mientras que una agenda organiza las actividades en el tiempo o una cruz se vehicula a determinadas creencias religiosas.

Se parte de que la identidad humana está vinculada a los fondos de conocimiento de las familias y comunidades, así como artefactos mediadores como una 
bandera, un himno nacional o un póster en la pared; recursos que nos informan sobre las creencias y recursos significativos de una determinada persona. En este sentido, la MMAE tiene el objetivo de analizar la identidad de los participantes a través de la detección de sus fondos de conocimiento, así como artefactos culturales significativos.

\section{Los talleres lúdico-reflexivos (TLR)}

El segundo método o estrategia aquí descrita para estudiar la identidad humana en ciencias sociales que aquí proponemos consiste en los talleres lúdicoreflexivos. Esta estrategia metodológica parte del hecho de que los grupos son espacios privilegiados de acercamiento al estudio de distintos fenómenos sociales como es el caso de la identidad. Aunque este método comparte características, desde el punto de vista metodológico, con la MMAE, el procedimiento es totalmente distinto ya que en este caso se trata de un nivel de análisis diferente dado que el sujeto puede no actuar igual en situación de grupo que de manera individual. En este sentido, los materiales colectivos que resultan de una dinámica grupal implican un conjunto de producciones y procesos colectivos, intersubjetivos y relacionales entre los participantes en el estudio. Käes (1976: 87) considera que cualquier grupo humano como objeto de estudio se representa por dos series de organizadores (esquemas subyacentes que organizan y subyacen a la actividad): los psíquicos (que tienen que ver con la subjetividad y las vivencias de los participantes) y los sociales o socioculturales (que tienen que ver con los contextos de los grupos, los condicionamientos históricos, físicos y simbólicos). Estos organizadores permiten, según el autor, comprender cualquier proceso grupal ya que subyacen a su dinámica. Las identidades sociales (nacionales, religiosas, lingüísticas, deportivas, étnicas) están relacionadas con procesos grupales y ciertas representaciones colectivas, por lo que el uso de una herramienta metodológica - en este caso los TLR - que implica lo grupal para el estudio de la identidad nos parece pertinente.

Al igual que en la MMAE, lo que se busca es facilitar procesos de reflexión alrededor de la identidad de los participantes, a pesar de que en este caso toma la forma de un «taller» como contexto de actividad a partir del cual generar o adaptar dinámicas vinculadas a la subjetividad e identidad de los miembros del grupo. El «taller», como herramienta metodológica, se ha utilizado en el ámbito educativo como estrategia didáctica o de trabajo cooperativo o colaborativo y desde el ámbito de la intervención social como instrumento para promover un determinado cambio. Lo que se propone aquí, fruto del trabajo de tesis doctoral de la primera autora que firma este trabajo, es considerar el «taller» como herramienta metodológica para estudiar la identidad humana.

En concreto, entendemos por TLR un espacio de trabajo grupal, desarrollado en sesiones de trabajo-juego-reflexión durante varios días, que se organiza a través de la combinación de un conjunto de herramientas con enfoque participativo (Geilfus, 1997), a través del uso de dinámicas y técnicas grupales integradas en 
función de un tema y objetivo central. Esta estrategia metodológica parte de dos fundamentos teóricos, a saber: los avances y desarrollos en psicología social de grupos, por un lado, y los enfoques participativos, por otro. Estos se ven reflejados tanto en el momento de reflexión incluido en la parte final de cada sesión de los talleres, por ser el espacio de construcción individual y colectiva del conocimiento y del saber sobre sí mismo y sobre los otros; como en lo referido a la aplicación de técnicas participativas, prioritariamente las dinámicas grupales, que propician la movilización de todo tipo de recursos de expresión (visuales, teatrales o de representación, movimiento corporal, moldeamiento, proyección, etc.), con las que la prioridad es que los participantes hagan actividades que propicien la reflexión sobre sus identidades. Una ventaja de este método, al igual que en el caso de la MMAE, es que de él siempre se desprende la generación de «documentos empíricos». Es decir, los talleres se caracterizan porque de ellos se espera obtener un producto tangible, en tanto que parte del supuesto pedagógico de que se aprende haciendo (Ander-Egg, 1991).

Partiendo de la noción de grupos de reflexión y grupos de discusión, a través de los cuales se materializan los procesos de elaboración de representaciones colectivas en torno a una determinada temática, así como se facilita la participación activa de los involucrados y la interpretación que hacen de lo acontecido (AnderEgg, 1990; Dellarossa, 1979; Montañés, 2009), proponemos los talleres a través de los cuales se desarrollan diversas dinámicas y actividades relacionadas con la identidad, dejando al final de cada sesión un momento de reflexión, sustentado en un «guión de debate» (Montañés, 2009) bastante abierto, que se inicia con la lectura de lo acontecido en el proceso grupal durante la sesión que le precedió.

Nuestra propuesta consiste en la secuenciación de dos talleres. El primero de ellos sirve para propiciar una reflexión sobre la propia identidad, conocer las auto y hetero-percepciones que tienen los participantes y para, en el ejemplo que presentamos, identificar y analizar los intereses de investigación y desarrollo profesional de los jóvenes estudiantes; mientras que el segundo taller se centra en el análisis, con ellos (ellas), de los resultados, profundizando en las identificaciones, en este caso, hacia la universidad y en la visión de futuro (correspondiente a la primer sesión), e incluyendo una parte formativa en términos de la enseñanza de la propia propuesta del método de los TLR (lo que se trabajó en la segunda sesión). Cabe aclarar que los ejemplos que aparecen en las técnicas utilizadas para los TLR se enmarcan en un estudio que tiene el propósito de analizar las percepciones que tienen, estudiantes indígenas, derivadas de su participación en un contexto institucional concreto, la Universidad Intercultural de Chiapas. En la tabla 2 se resumen las actividades o tareas desarrolladas, clasificadas a partir del tipo de técnica en que se inscribe cada una. Para el diseño de un TLR es necesario combinar los cuatro tipos de técnicas (Basagoti y Bru, 2002) de manera creativa, puesto que como se verá más adelante donde se describe cada una, de esta combinación depende que se establezcan las condiciones propicias para el proceso narrativo de la propia identidad y el despliegue de la subjetividad de los participantes.

EMPIRIA. Revista de Metodología de Ciencias Sociales. N. ${ }^{\circ}$ 26, julio-diciembre, 2013, pp. 175-200. ISSN: 1139-5737, DOI: 10.5944/empiria.26.7157 
Tabla 2. Tipo de técnicas y Actividades de los dos Talleres Lúdico-Reflexivos (TLR)

\begin{tabular}{|c|c|c|c|}
\hline De Iniciación & De Cohesión & $\begin{array}{c}\text { De Producción } \\
\text { Grupal }\end{array}$ & $\begin{array}{l}\text { De Evaluación- } \\
\text { Reflexión }\end{array}$ \\
\hline $\begin{array}{l}\text { «Historia de su nom- } \\
\text { bre» Taller 1, Sesión } 1 \\
\text { (T1S1) } \\
\text { «La foto en la mano» } \\
\text { T1S1 } \\
\text { «Nombre y movi- } \\
\text { mientos», Taller 1, } \\
\text { Sesión } 2 \text { (T1S2) } \\
\text { «Mi reflejo en los } \\
\text { otros», Taller 1, Se- } \\
\text { sión } 3 \text { (T1S3) } \\
\text { «Globos con abrazo», } \\
\text { Taller 2, Sesión 1 } \\
\text { (T2S1) } \\
\text { «Pantomimia e ima- } \\
\text { gen de semejanzas», } \\
\text { Taller 2, Sesión } 2 \\
\text { (T2S2) }\end{array}$ & $\begin{array}{l}\text { «Yo soy» T1S1 } \\
\text { «Yo no soy» T1S1 } \\
\text { «Cinta de Prejuicios» } \\
\text { T1S2 } \\
\text { «Reconociéndonos en } \\
\text { imágenes» T1S3 } \\
\text { «Reloj», T2S1 } \\
\text { «Competencia o Co- } \\
\text { laboración», T2S1 }\end{array}$ & $\begin{array}{l}\text { «Líneas de vida» } \\
\text { T1S2 } \\
\text { «Árbol de vida y rela- } \\
\text { ciones con los otros» } \\
\text { T1S2 } \\
\text { «Roles y etiquetas. Lo } \\
\text { fuimos, somos y sere- } \\
\text { mos» T1S2 } \\
\text { «Mapas Colectivos» } \\
\text { T1S3 } \\
\text { «Nosotros los de la } \\
\text { UNICH» T1S3 } \\
\text { «El camino», T2S1 } \\
\text { «Lo que un día fui, } \\
\text { lo que soy y lo que } \\
\text { seré», T2S1(dibujo } \\
\text { identitario) } \\
\text { «Historietas juveni- } \\
\text { les», T2S1 } \\
\text { «Lluvia de ideas y } \\
\text { dramatización», T2S1 } \\
\text { «Historias Múltiples», } \\
\text { T2S1 } \\
\text { «Lluvia de ideas con } \\
\text { tarjetas», T2S2 } \\
\text { «Papelógrafos: Pla- } \\
\text { neación de un taller» } \\
\text { y «En río Revuelto } \\
\text { ganacia de pescado- } \\
\text { res», T2S2 }\end{array}$ & $\begin{array}{l}\text { «Reflexión co- } \\
\text { lectiva» T1S1 } \\
\text { «Reflexión co- } \\
\text { lectiva» T1S2 } \\
\text { «Moldeándonos } \\
\text { en barro» T1S3 } \\
\text { «Reflexión co- } \\
\text { lectiva» T1S3 } \\
\text { «Reflexión co- } \\
\text { lectiva», T2S1 } \\
\text { «Evaluación y } \\
\text { cierre del taller», } \\
\text { T2S2 }\end{array}$ \\
\hline
\end{tabular}

\section{TÉCNICAS PARA EL ESTUDIO DE LAS IDENTIDADES}

A continuación se ilustran algunas técnicas vehiculadas a la MMAE y a los TLR. La ejemplificación, con casos empíricos, de los distintos instrumentos o estrategias nos permite describir su modo de aplicación, así como observar algunos posibles resultados de su utilización como recurso metodológico.

Los ejemplos que aparecen en el apartado dedicado a la MMAE están extraídos de una línea de investigación que tiene el objetivo de vincular familia-escuela en contextos con elevada diversidad cultural a través de la detección de los fondos de conocimiento, formas de vida y artefactos de los alumnos y familias por parte de un maestro/a (Esteban-Guitart, Oller y Vila, 2012). La intención es 
modificar su práctica educativa partiendo de la información documentada, que pasa a convertirse en recursos pedagógicos que permiten vincular el curriculum con la vida de los estudiantes (sus conocimientos previos, su fondo social, familiar y cultural). En el segundo caso, las técnicas que ilustran el método de los TLR, tal y como se ha mencionado anteriormente, se realizan como parte de un trabajo de tesis doctoral en curso que tiene el objetivo de analizar el impacto en las identidades que tiene participar en un modelo educativo universitario intercultural por parte de jóvenes indígenas de Chiapas.

\section{Técnicas asociadas a la multi-metodología autobiográfica extendida}

En la tabla 1, tal y como hemos indicado anteriormente, aparecen las técnicas propuestas al investigador que, en función de su pregunta de investigación y estudio, puede elegir. El objetivo es detectar los fondos de conocimiento, las formas de vida y la identidad de los participantes que se convierten en co-investigadores al participar activamente en el proceso de creación-recogida de información de naturaleza autobiográfica. A continuación se describen, brevemente, las quince técnicas, ilustrando el resultado de la aplicación de alguna de ellas.

El primer bloque de técnicas de la MMAE agrupa tres modelos de entrevista. La primera, tomada de McAdams (2003), consiste en una entrevista abierta tipo historia de vida. Se trata, en definitiva, de construir un relato sobre la vida de uno mismo a partir de distintas preguntas que el investigador plantea. El autor de la propuesta, McAdams (2003), parte de la creencia según la cual la identidad humana es una historia de vida, toma la forma de relato sobre uno mismo, con sus peculiares tramas, y en la que aparecen distintas escenas y personajes. «En mi teoría de la identidad en tanto que historia de vida, he argumentado que la identidad toma la forma de historia, con escenas, ambientes, personajes, objetivos y temas» (McAdams, 2003: 187). En realidad, hay toda una línea de investigación (Bruner, 2003; Esteban-Guitart, Nadal y Vila, 2008) que considera la identidad como un producto o fenómeno narrativo y, por lo tanto, sujeto a las características de una narración: trama o argumento, obstáculo o problemas, reflexión ante la amenaza o suceso, influencia del pasado y las voces culturales que uno internaliza — se apropia-, cambio derivado del intento de solución y vuelta a la situación no problemática. «Es mediante nuestras propias narraciones como principalmente construimos una versión de nosotros mismos en el mundo, y es a través de sus narraciones como una cultura ofrece modelos de identidad y de acción a sus miembros» (Bruner, 1997: 15). La entrevista de McAdams, disponible en la pagina web del «Foley Center for the study of Lives» http://www.sesp. northwestern.edu/foley/instruments/interview/, pretende generar una historia de vida recuperando los capítulos o momentos vitales más importantes para la persona, el episodio más triste, el más feliz, el mayor reto que uno ha tenido que enfrentarse o la ideología personal.

En el caso de la entrevista «Fondos de conocimiento», se trata del instrumento metodológico utilizado en un proyecto desarrollado en la Universidad de 
Arizona (EE.UU) (González, Moll y Amanti, 2005). El objetivo de la entrevista es detectar los fondos de conocimiento que poseen las familias y, para ello, se realizan preguntas alrededor de su historia laboral y educativa. En concreto, en el proyecto original se proponía hacer tres visitas a las familias, con tres entrevistas asociadas. En la primera se pregunta por la historia familiar y laboral de los participantes. En la segunda se centra la discusión alrededor de las prácticas, actividades, que realizan en casa, así como las rutinas. En la última entrevista se tratan temas educativos como su experiencia escolar, prácticas de alfabetización que realizan o las lenguas que usan y bajo qué circunstancias.

Finalmente, el tercer modelo de entrevista propuesto en la MMAE consiste en el desarrollo de cinco cuestiones extraídas del trabajo de Durand (2011). Cuestiones vinculadas a la percepción que tienen los padres de sus hijos, el rol paterno/materno, las relaciones con sus hijos y, finalmente, las relaciones con la escuela. Vinculado al tema de los fondos de conocimiento, en este caso se trata de detectar las creencias que las personas adultas tienen en relación a la educación y a su maternidad/paternidad.

El segundo bloque de técnicas propuestas bajo el nombre de «tarea del dibujo identitario revisada» incluye el «dibujo identitario» («self portrait») desarrollado por Bagnoli (2004), un listado de definiciones identitarias y la tarea de las diez definiciones que sigue la propuesta de Hartley (1970). En el primer caso, los participantes deben hacer un dibujo sobre sí mismos siguiendo la instrucción: «Podrías intentar dibujar quién piensas que eres en este momento. Puedes añadir, si quieres, las personas y cosas (actividades, instituciones) que para ti sean más importantes en este momento». Una vez obtenido el dibujo, el participante explica su contenido y el investigador aprovecha para realizar preguntas con el objetivo de generar narrativas identitarias por parte del entrevistado. En la figura 2 se puede ver un ejemplo de dibujo identitario de una chica de 18 años. En este caso, la participante dice: «me he dibujado dentro de un círculo negro que me bloquea, que no me deja salir y a la vez tampoco deja entrar a nadie, representa mi espacio vital. En mi cabeza hay un interrogante que expresa el enredo que tengo. El color verde del cuerpo expresa la esperanza de una salida que se expresa con el color amarillo de fuera del círculo». A partir de aquí, el entrevistador puede realizar una entrevista con la participante con el objetivo de profundizar en el contenido que aparece en el dibujo. Cabe aclarar que a pesar de que se trata de una tarea proyectiva, aquí se plantea como una tarea descriptiva de los contextos de actividad, así como recursos que uno utiliza para autodefinirse, más que una supuesta vía de entrada al inconsciente. En este sentido, esta técnica o herramienta metodológica se ha utilizado para analizar la influencia de la cultura en el autoconcepto (Esteban-Guitart y Vila, 2010; Esteban-Guitart, Nadal, Vila y Rostan, 2008), para observar los cambios en la identidad personal a lo largo de la vida, según un diseño transversal (Gifre, Monreal y Esteban-Guitart, 2011), así como para detectar fondos de conocimiento e identidad con el objetivo de vincularlos a la creación de unidades curriculares específicas (Saubich y EstebanGuitart, 2011).

EMPIRIA. Revista de Metodología de Ciencias Sociales. N. ${ }^{\circ}$ 26, julio-diciembre, 2013, pp. 175-200. ISSN: 1139-5737, DOI: 10.5944/empiria.26.7157 


\section{Figura 2. Ejemplo de dibujo identitario}

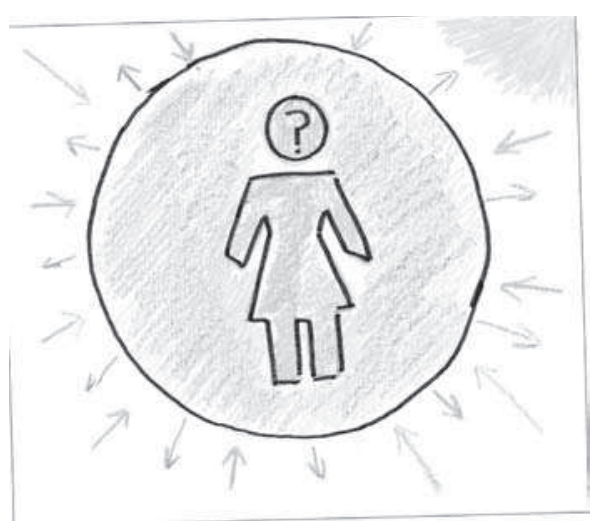

En relación a la tarea de las definición identitaria el participante debe responder a la siguiente pregunta: «Imagínate que quieres presentarte a una persona que no te conoce, ¿cómo lo harías?, ¿cómo te definirías a ti mismo/a?». Finalmente, en la tarea de las diez definiciones, el entrevistado debe responder, mediante adjetivos o frases — diez veces—, a la cuestión «Yo soy:». Además, los participantes deben ordenar las diez autodefiniciones según consideren ser más importantes en la auto-descripción.

El tercer bloque de técnicas lleva por título «detección de artefactos, rutinas y formas de vida». En esta ocasión se proponen cuatro técnicas al investigador. En el diario personal de una semana, basado en la propuesta original de Bagnoli (2004), el participante debe elaborar, durante siete días, un sencillo diario en el que anota la actividad o cosa más importante que ha realizado al cabo del día, con quién ha estado y con quién ha pensado. En el caso de la técnica «detección de artefactos», pensada a partir de la propuesta de González-Patiño (2011), los participantes anotan aquellos artefactos o instrumentos culturales que para ellos son más significativos y están incrustados en sus formas de vida como, por ejemplo, el caso de la televisión, un determinado libro (la Biblia, por ejemplo) o un póster de un artista colgado en la habitación. Además de situar los distintos artefactos, las personas ponen en otras dos columnas el sitio que están, así como el uso asociado al mismo. Por ejemplo, una televisión que está en una habitación o dormitorio y sirve para entretenerse, o un libro sagrado, el Corán, que está en el comedor y que una determinada familia lee cada día para acompañar su plegaria. Finalmente, las dos últimas técnicas de este bloque utilizan fotografías con el objetivo de analizar las rutinas y formas de vida de los participantes. En este sentido, Poveda, Casla, Messina, Morgade, Rujas, Pulido y Cuevas (2007) han utilizado un procedimiento a partir del cual los participantes deben traer fotografías representativas de sus rutinas durante una semana para ser, posteriormente, discutidas con el investigador. Aquí proponemos una variante con el objetivo de detectar las rutinas educativas. Se trata de que la familia o participante en el 
estudio debe traer, un mínimo orientativo de 5 fotografías por día, en las que considere que está en una situación educativa. Ello permite analizar qué entiende por educación el entrevistado, así como las prácticas de alfabetización y educación de una determinada persona o familia. En la figura 3 se puede observar un ejemplo de fotografía, rutina educativa, en la que un padre está sentado con su hijo mostrándole alguna cosa a través de un ordenador portátil.

Figura 3. Ejemplo de rutina educativa captada a través de una fotografía

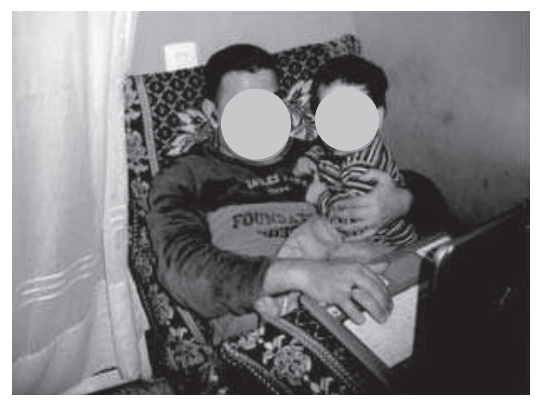

Finalmente, en el último bloque de técnicas llamado «mapas psicológicos» o «psicogeomapas», se incluyen un total de cinco herramientas que, a través de una determinada representación, transmiten información sobre los principales hechos vitales de una persona a lo largo de su biografía (el caso del «cronograma»), los miembros de una familia, mínimo tres generaciones, y sus relaciones (el «genograma»), las relaciones de la familia o persona con su entorno (el «ecomapa», Hartman, 1979), específicamente con el entorno geográfico (el «geomapa») o una representación sobre las personas, actividades y cosas significativas para una persona (el «círculo significativo»).

Figura 4. Ejemplo de «círculo significativo»

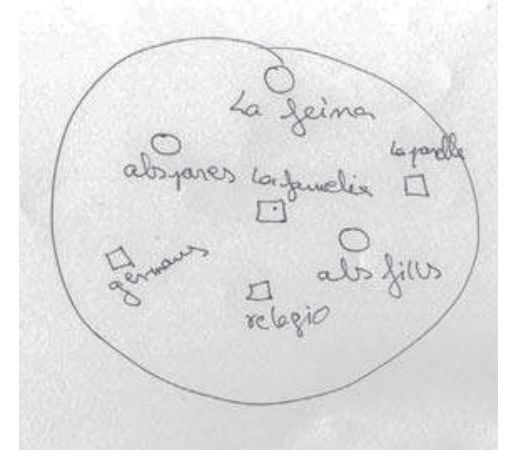

En la figura 4 se ilustra el resultado de la técnica «círculo significativo». La instrucción es la siguiente: «Dibuja un círculo y pon en el medio un pun- 
to. Imagínate que este círculo eres tú. Dentro puedes poner, representado por círculos más pequeños, las personas que sean más significativas, importantes, para ti. En cuadrados puedes representar las actividades, aficiones, instituciones que sean más importantes para ti. Piensa que como más al centro pongas los círculos y los cuadrados, eso significa que más valorados, más relevantes, son». En este caso, la persona entrevistada, una madre de familia procedente de Marruecos que vive en Catalunya, ha representado, como personas significativas, la familia («la familia»), su pareja («la parella»), sus hijos («als fills»), sus padres («als pares») y sus hermanos («germans»). Como actividades, instituciones o cosas ha dibujado la religión («religió») y su trabajo («la feina»). En el dibujo se observa claramente como la protagonista ha situado en el medio del círculo a la familia. A partir de aquí, el investigador puede preguntar, con el objetivo de obtener más información, y el entrevistado explicar, su «círculo significativo».

Figura 5. Ejemplo de «geomapa»

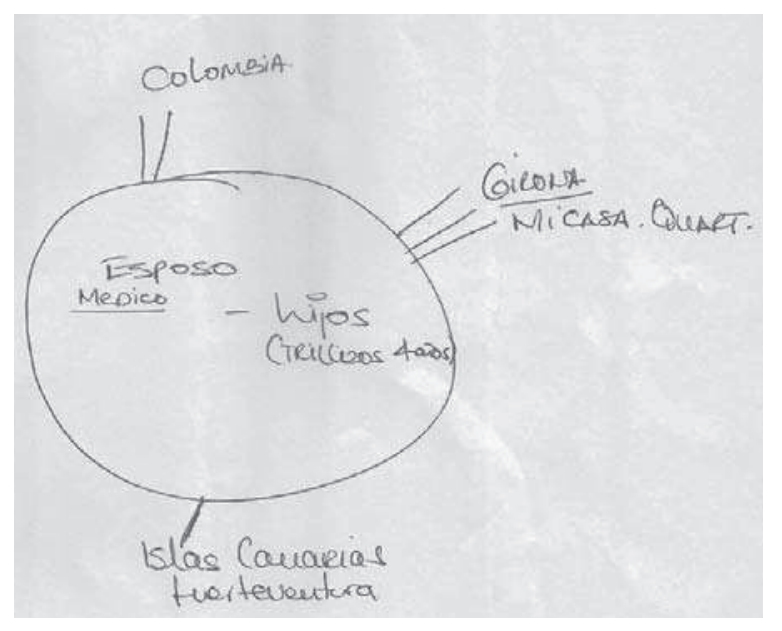

En el «cronograma», el participante sitúa, en una línea temporal, aquellas fechas y hechos más importantes, más significativos, de su vida hasta el momento. Uno puede señalar, por ejemplo, la fecha de su boda, de la muerte de un ser querido o del cambio de trabajo. En el caso del «genograma», la persona representa los miembros de su familia en, como mínimo, tres generaciones, así como sus relaciones de parentesco, pudiendo aparecer otras informaciones como enfermedades. Por lo que hace al «ecomapa» y al «geomapa» la estructura y el procedimiento es similar. La persona, en el primer caso, establece las relaciones entre su contexto familiar y otros contextos sociales que rodean a la familia y que son importantes, ya sea positiva o negativamente. Por ejemplo, la familia extensa, el trabajo, la educación, los servicios de salud, la religión o instituciones deportivas. La particularidad de esta representación es que se 
debe conectar la familia con los otros sistemas o contextos a través de líneas que pueden significar «relación estresante» (una línea en zig-zag), «relación, vínculo» (una línea), «relación, vínculo, intenso» (dos líneas), «relación, vínculo, muy intensa» (tres líneas) dependiendo del modelo de codificación. El «geomapa» que aquí presentamos es una derivación en la que el participante relaciona la familia con los entornos geográficos como un país, un paisaje, una ciudad o un pueblo. En el caso del ejemplo que aparece en la Figura 5 se puede observar como aparece un país, Colombia, una ciudad, Girona, y un archipiélago, las Islas Canarias, concretamente Fuerteventura. La relación más intensa es con Girona, seguido de Colombia y las Islas Canarias. Como en las otras técnicas y procedimientos, la representación gráfica es una excusa para generar información, en este caso, vinculado a temas como la transnacionalidad en inmigrantes, personas que proceden de un contexto nacional determinado y residen en otro.

\section{Técnicas utilizadas en los talleres lúdico-reflexivos}

Para la organizar las actividades o tareas de los TLR se consideraron tres momentos o etapas que se construyeron considerando los tipos de técnicas grupales que identifican Basagoiti y Bru (2002). Las actividades incluidas en los dos TLR fueron inventadas o adaptadas de técnicas ya existentes, algunas son forman parte de la propuesta de la MMAE. Las referencias bibliográficas que se usaron para la definición de las actividades fueron las siguientes: Ander-Egg (1990), Antunes (1989), Candelo, Ortiz y Unger (2003), Chapela (2005), Ferreira y Pasut (1998).

El primer momento refiere a la creación de ambiente propicio, en él son indispensables las técnicas de iniciación, que son las que posibilitan las condiciones necesarias para que se genere confianza, conocimiento entre participantes y desinhibición (Basagoiti y Bru, 2002), y las de cohesión, que buscan generar cierta integración en el grupo, necesaria para la circulación de imaginarios y discursos colectivos, permitiendo la lectura de proceso basada en los organizadores grupales (Käes, 1976). En ambos casos, se trata de preparar al grupo para entrar en el tema central, segundo momento, por lo que las tareas programadas deben girar en torno al tema, aunque sea en un nivel superficial. Para ejemplificar esto tomemos el caso de «Historia de su nombre», dinámica en la que los participantes escribían en un globo su nombre, se juega con los globos dividiendo a los participantes en dos equipos y dando diversas indicaciones se hacen desplazamientos por el espacio (ver figura 6), de manera que al final se formen parejas con la finalidad de que platiquen la historia del nombre que tienen (¿por qué les pusieron ese nombre? ¿A qué se debe?) y cuando terminan presentan en plenaria la historia de la (el) participante con quien hicieron la actividad. 
Figura 6. «Historia de su nombre»

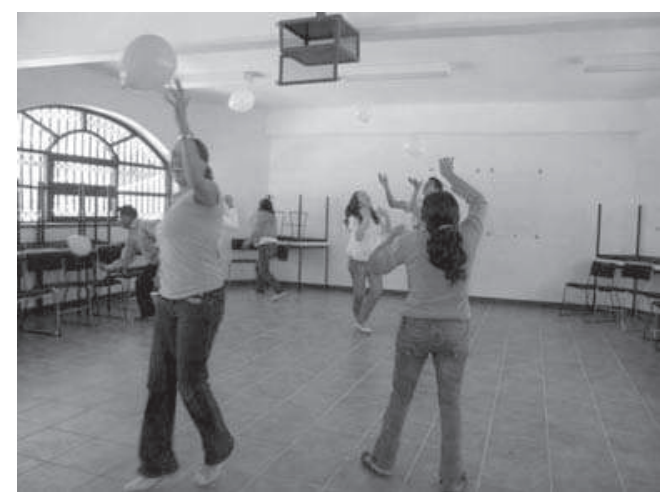

En el caso de las técnicas de cohesión, se adaptó la «tarea de las diez definiciones» de los dibujos identitarios, pidiendo a los participantes que elaboraran una lista con 20 definiciones del «yo soy» (Hartmann, 1979) y al terminar marcaban las tres que más los identificaban y las tres que más les disgustan. Luego se da la indicación «Elijan con la mirada, sin hablar a la persona con la que te gustaría compartir lo que escribiste. Ya que la hayas elegido, ve a buscarla, no esperes a que te elijan, busca tú con quien quieras compartirlo», para finalmente con todo el grupo comentar las seis definiciones elegidas y analizar colectivamente similitudes y diferencias.

Figura 7. «Yo soy»

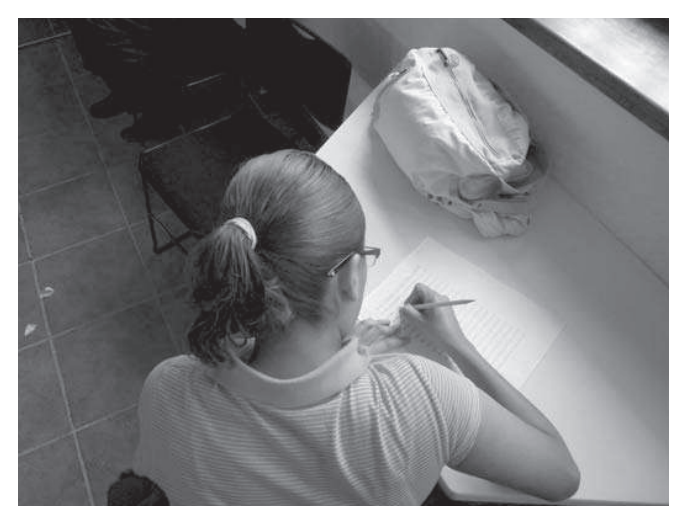

Una vez que se considera que el ambiente es propicio para un buen trabajo grupal, lo que puede determinarse a través de la observación participante, intuición y experiencia del investigador, se pasa al segundo momento el tema central, que es la etapa donde se enfatiza en el hacer, vivenciar, generar expresión a través de acciones y actividades relacionadas directamente con las preguntas centrales y 
propósitos de la investigación. En este momento las técnicas de producción grupal son el eje para el diseño metodológico, porque facilitan el cumplimiento de las tareas centradas en un tema particular y ayudan a organizar al grupo. Para este caso ejemplificamos dos dinámicas «Árbol de vida y relaciones con los otros» y «Lluvia de ideas y dramatizaciones». En el caso de la primera, su realización efectiva se desprende de la actividad «Líneas de vida», en la que los participantes identifican los momentos más significativos de su historia de vida y los organizan cronológicamente en etapas a las que les ponen un nombre. Al terminar se pasa a la elaboración de los árboles de vida, cuyo objetivo es que los participantes elaboren una representación gráfica de su historia de vida, partiendo de su identidad personal, y construyendo sus relaciones con los otros. Para la elaboración de los árboles se pide que tracen un árbol con los siguientes elementos: raíces, tronco, ramas y follaje u hojas, se les explica que estos elementos están relacionados con las etapas de la actividad anterior: la raíz y tronco con lo que fueron en su infancia $y$ en el pasado (hace 5-6 años); las ramas a lo que son actualmente (presente); y el follaje a lo que desean ser (futuro). En la figura 8 puede verse un ejemplo de árbol de vida, elaborado de acuerdo a la indicación dada en el taller: en la raíz del lado izquierdo lo que siempre serán y del lado derecho las personas o grupos con las que siempre se relacionarán e identificarán; en el tronco, lado izquierdo lo que fueron y ya no son y del derecho las personas o grupos con las que se identificaron o relacionaron pero que ya no lo hacen; en las ramas, lo que son del izquierdo y las personas y grupos con las que se relacionan o identifican en la actualidad del derecho; por último, en el follaje lo que quieren o desean ser del izquierdo y las personas con las que les gustaría relacionarse, identificarse y trabajar del derecho. $\mathrm{Al}$ terminar cada participante presenta su árbol y lo explica.

Figura 8. «Árbol de vida»

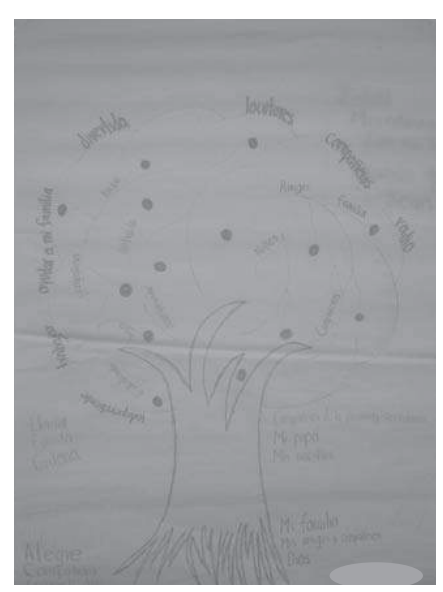

Como puede apreciarse esta actividad combina y recrea las técnicas del dibujo identitario, particularmente los círculos significativos, y los mapas psicológi- 
cos o geomapas, particularmente el cronograma, ambas propuestas de la MMAE que ya hemos explicado.

El segundo ejemplo corresponde a la tarea de «Lluvia de ideas y dramatizaciones», técnica con la que se buscó trabajar la identidad hacia el espacio escolar en el que estudian, así como las influencias de este en la construcción identitaria, y la representación de futuro de sí mismos con relación al ámbito profesional. Para esto se recuperaran con la técnica de «Lluvia de ideas» (Brainstorming) las respuestas que da el grupo a seis preguntas, tres para cada tema (identificación hacia la UNICH y visión de sí mismo en el ámbito profesional). Se conforman dos equipos libremente y se pide que cada uno haga una representación (dramatización) a partir de las respuestas que dieron. Se presentan las dramatizaciones y se graban en video con el permiso de los participantes. La figura 9 ilustra extractos del video de las dos dramatizaciones que editaron los mismos participantes.

Figura 9. «Dramatizaciones»

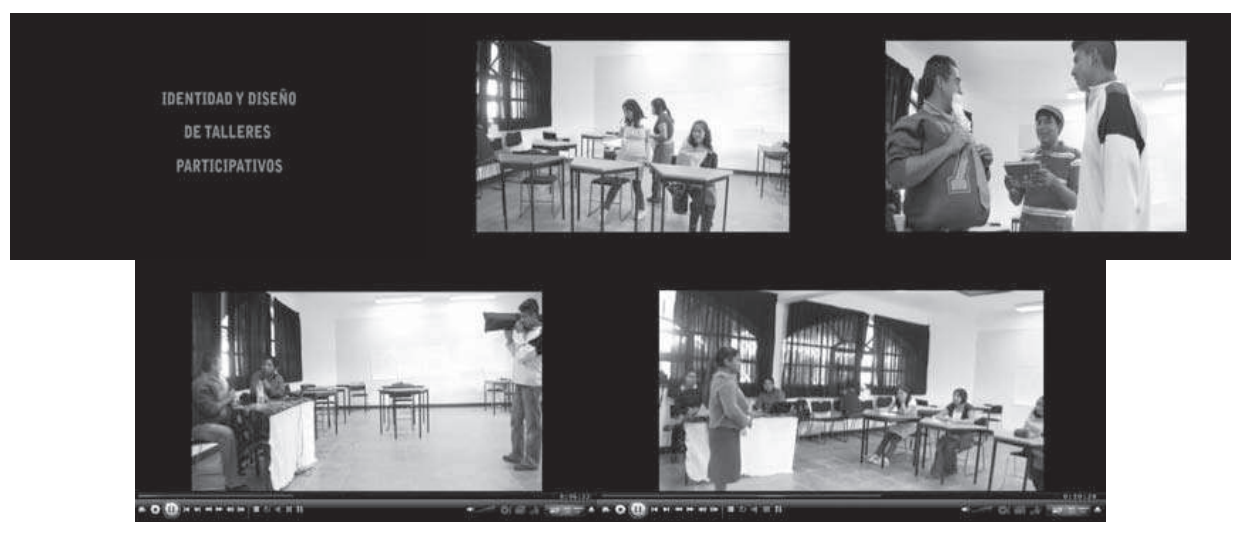

La riqueza del empleo de las dramatizaciones radica en que, por un lado, ayuda a comprender las representaciones sociales de los colectivos, central en el estudio de las identidades; $y$, por el otro, posibilita la permanente interacción con los otros y jugar con todos los roles para mirar los fenómenos desde otro lugar (Paz, 1996).

Finalmente, el tercer momento de la propuesta combina metacognición y evaluación. La primera se refiere a las reflexiones que desarrolla el grupo sobre el propio proceso, es decir, compartir y poner en común, cómo recupera y significa las vivencias de cada actividad, se explicita lo aprendido, lo sentido, lo pensado y la manera en que lo hace. En el caso de los TLR en la última sesión se combinaba el momento de reflexión con el de evaluación proponiendo alguna técnica en particular para ello y desde un enfoque participativo, es decir, todos los participantes y facilitadores del taller toman la palabra para valorar el proceso. Para esta etapa del proceso se emplean las técnicas de medición, o mejor dicho de evaluación y reflexión, que son aquellas que sirven para conocer cómo 
son las interacciones dentro del grupo e identificar el impacto del trabajo desarrollado, así como de los facilitadores y participantes en el proceso (Basagoiti y Bru, 2002).

Una actividad usada para este propósito fue «Moldeándonos en barro», como lo ilustra la figura 10 se pide a los participantes que elaboren una figura que represente lo que significó para ella (él) el grupo y en sí el taller. Además, se ponen dos peguntas que deben pasar a responder de manera individual con una palabra o frase cada uno, sobre lo aprendido y lo que dejan de sí mismos. Al terminar se pasa al momento de reflexión, que se hace siempre en plenaria, y procurando organizar el espacio de manera que fomente la comunicación horizontal (todos viéndose de frente y de preferencia sin objetos entre las personas, como bancas o mesas).

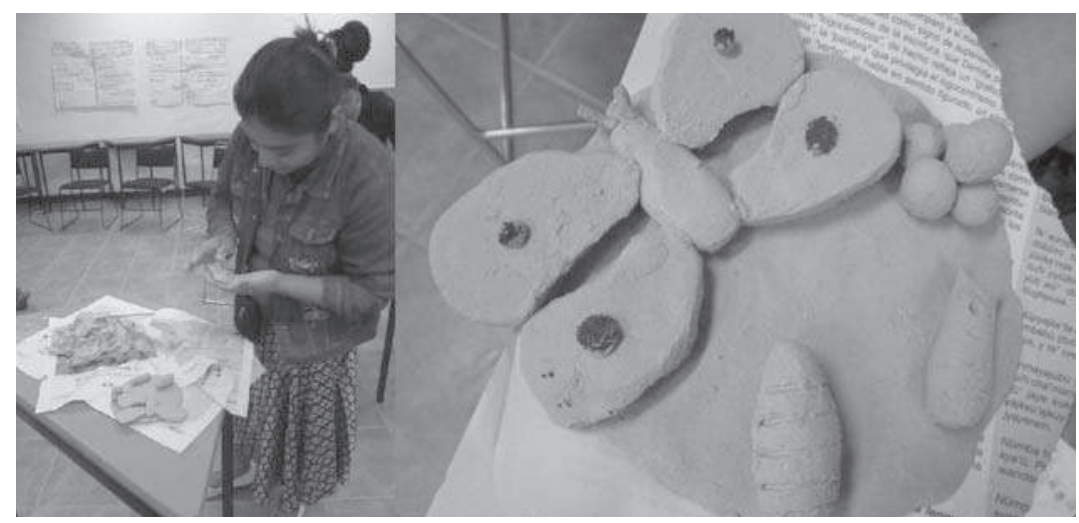

Uno de los testimonios que se obtuvieron con esta técnica fue el siguiente: «( $($ ) una metamorfosis, ( $)$ entrando al taller me sentí como encerrada, yo sola, ( $($ ) puedes estar rodeada de mucha gente y al mismo tiempo sentirte sola,

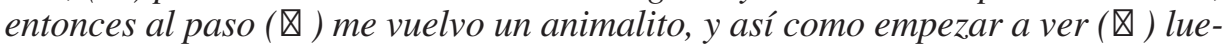
go me enclaustré en el taller $(\mathbb{\nabla})$ me sentí dentro de un capullo $(\mathbb{\nabla})$ llevo de este

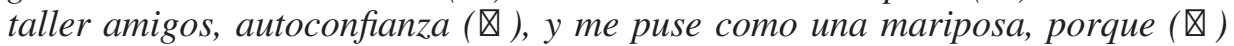
representa poder volar como las aves ( $\nabla$ ) una metamorfosis, un cambio, porque sentí realmente que sí conocí, y dije bueno si éste es el motivo de taller en qué me ayuda para seguir hacia adelante, y para que me ayude también a conocer diferentes ideas, diferentes formas de analizar la situación y ( $\mathrm{Q}$ ) poder en lo externo aplicar este conocimiento con los demás, darle también la enseñanza porque no quiero que sólo se quede aquí».

Como puede intuirse, de los materiales que se producen en los TLR los más útiles para el trabajo de análisis e interpretación de un problema de investigación son los que se desprenden de las técnicas de producción grupal y los de evaluación-reflexión, aunque tanto los de iniciación, como los de cohesión arrojan productos y evidencias que pueden ser retomadas en la elaboración de argumentos sobre el problema de investigación. 


\section{CONCLUSIÓN}

Este trabajo ha ilustrado dos estrategias metodológicas para investigar las identidades en ciencias sociales, a saber, la multi-metodología autobiográfica extendida y los talleres lúdico-reflexivos. En concreto, se han ejemplificado distintas técnicas asociadas a cada uno de estas estrategias cualitativas. Tanto la MMAE como los TLR son métodos participativos a través de los cuales los entrevistados se convierten en protagonistas activos en la recogida y generación de información empírica. En este sentido, son «co-investigadores» (Bagnoli, 2004) ya que realizan alguna actividad, más allá que responder un cuestionario o unas preguntas semi-estructuradas. En el caso de la MMAE, por ejemplo, realizan un dibujo sobre sí mismos, realizan y recogen fotografías, así como representan su relación con aquello que les rodea. En los TLR también dibujan, el caso de los árboles, y participan en distintas actividades con el objetivo de generar una reflexión alrededor de cuestiones identitarias.

Pensamos que son herramientas metodológicas que permiten estudiar empíricamente las «crónicas del yo», es decir y, recuperando la definición de Giddens (1997): «relato o relatos por los que tanto el individuo en cuestión como los demás entienden reflejamente la identidad del yo» (p. 293).

En nuestra opinión, el principal beneficio de ambas estrategias es que permiten profundizar en la información que uno obtiene mediante la utilización de estrategias cuantitativas como, por ejemplo, un determinado cuestionario sobre «identidad étnica». Además, estas herramientas promueven el «uso de comunicación diversificada», trascendiendo el uso del lenguaje oral, para experimentar la expresión artística (a través de los dibujos y el barro, por ejemplo), el empleo de recursos teatrales y de movimiento corporal, así como la generación de lenguaje metafórico para expresar ideas. La información que se obtiene es rica y permite captar los sentidos y significados identitarios en primera persona. Es decir, se documentan y analizan productos, artefactos, realizados por los participantes que pasan a convertirse en auténticos «documentos de vida» o «textos identitarios» que el investigador, a partir de su pregunta de investigación y marco teórico, interpreta.

A través de estas herramientas, la sensibilidad y apertura de los participantes al aprendizaje, los lleva a un «ámbito personal». Esto se puede interpretar como que se genera una comprensión reflexiva, es decir, se accede al proceso de construcción reflexiva de las interpretaciones de los sujetos sobre sí mismos. Este ámbito tiene diferentes niveles descritos por los sujetos, uno de ellos se refiere al descubrirse, aceptarse, resignificar momentos dolorosos y elaborarlos para que no sigan siendo tanto, a través de la oportunidad de expresar, recordar y hablar de ellos. A pesar de que la utilización que aquí se ha descrito no es con fines psicoterapéuticos sino vinculados a la investigación alrededor de la identidad.

A pesar de los potenciales beneficios que pensamos tiene el uso de metodologías, como las descritas, es importante señalar sus limitaciones. Como mínimo queremos hacer referencia a la dificultad de analizar el material empírico sin un ajustado marco teórico que oriente la interpretación de los resultados hallados.

EMPIRIA. Revista de Metodología de Ciencias Sociales. N. ${ }^{\circ}$ 26, julio-diciembre, 2013, pp. 175-200. ISSN: 1139-5737, DOI: 10.5944/empiria.26.7157 
Dada la complejidad del corpus textual que se genera, auténticos productos identitarios (generados por los participantes), los propios sujetos deben participar en el análisis de los datos en el sentido de que el investigador debe volver a ellos para precisar, cuando sea necesario, el sentido y significado del producto. Ello puede generar dificultades en el transcurso del análisis e interpretación ya que se convierten en acciones en cíclicas y en paralelo que, a la vez, se retroalimentan e intercalan.

Precisamente dada la naturaleza personal del material empírico que se genera, nos parece pertinente terminar el artículo enfatizando la necesidad del investigador de negociar con los participantes en el estudio la naturaleza y uso del material, así como «devolver» cualquier producto (por ejemplo un artículo) a los entrevistados. En este sentido, el proceso de co-investigación (investigadorparticipantes) se convierte en un camino de ida y vuelta en el que se parte de los entrevistados, proceso de investigación, para terminar con ellos, compartiendo los resultados obtenidos, así como las interpretaciones realizadas. Algo que caracteriza cualquier investigación, en general, pero que debe tenerse especialmente en cuenta en estudios cualitativos, en particular.

\section{BIBLIOGRÁFIA}

ALCOFF, L. (1989): «Feminismo cultural versus pos-estructuralismo: la crisis de identidad en la teoría femenina», en: Feminaria, Año II, No. 4, noviembre de 1989, pp. 1-18, Buenos Aires, Tejepalabras.

ANDER-EGG, E. (1990): Reprensando la Investigación-Acción-Participativa, Argentina, Lumen.

ANDER-EGG, E. (1991): El taller. Una alternativa para la renovación pedagógica, Argentina, Magisterio del Río de la Plata.

ANTUNES, C, (1989): Manual de técnicas de dinámica de grupo, de sensibilización y lúdico-pedagógicas, Lumen, Argentina.

BAGNOLI, A. (2004): «Researching identities with multi-method autobiographies», Sociological Research Online, 9, disponible en http://www.socresonline.org.uk/9/2/ bagnoli.html [consulta: 10-4-2008]

BAGNOLI, A. (2009): «Beyond the standard interview: the use of graphic elicitation and arts-based methods», Qualitative Research, 9, pp. 547-570.

BASAGOITI, M, y BRU, P. (2002): «Mira quién habla» (El trabajo con grupos en la I.A.P.)», en: VILLASANTE, T. R., et. al. (Coords.) (2002), La investigación social participativa. Construyendo ciudadanía, El Viejo Topo - Red de Colectivos y Movimientos Sociales (CIMS), pp. 125-142.

BAUMAN, Z. (2000): Liquid modernity, London, Blackwell Publishers.

BAUMAN, Z. (2003): Comunidad: en busca de seguridad en un mundo hostil, Madrid, Siglo XXI.

BECK, U. (1998): La sociedad del riesgo. Hacia una nueva modernidad, Barcelona, Paidós.

BRUNER, J. (1997): La educación. Puerta de la cultura, Madrid, Visor.

BRUNER, J. (2003): La fábrica de historias. Derecho, literatura y vida, Buenos Aires, Fondo de Cultura Económica de Argentina.

EMPIRIA. Revista de Metodología de Ciencias Sociales. N. ${ }^{\circ}$ 26, julio-diciembre, 2013, pp. 175-200. ISSN: 1139-5737, DOI: 10.5944/empiria.26.7157 
CASTELLS, M. (1999): La era de la información: El poder de la identidad, Volumen II, México, Siglo XXI.

CANDELO, C, ORTIZ, G, y UNGER, B (2003): Hacer talleres. Una guía práctica para capacitadores, WWF-DSE-IFOK, Colombia, Disponible en http://www.gwpsudamerica.org/docs/publicacoes/doc_123_sp.pdf [consulta: 16-3-2009]

CHAPELA, L. M, (2005): Relaciones interculturales. Cuaderno de trabajo, FOMEIMCGEIB -SEP, México.

COLE, M. (1996): Cultural psychology: a once and future discipline, Londres, Harvard University Press.

COOLEY, C. (1902): Human nature and the social order, New York, C. Scribner's sons.

DELLAROSA, A. (1979): Grupos e instituciones, Paidós, Argentina.

DUBAR, C. (2002): La crisis de las identidades: la interpretación de una mutación, Barcelona, Bellaterra.

DURAND, T. M. (2011): «Latina mothers cultural beliefs about their children, parental roles, and education: implications for effective and empowering home-school partnerships», The Urban Review. Issues and Ideas in Public Education, 43, pp. 255278.

ERIKSON, E. H. (1950): Childhood and society, New York, Norton.

ESTEBAN-GUITART, M. (2010): Geografías del desarrollo humano. Una aproximación a la psicología cultural, Barcelona, Aresta.

ESTEBAN-GUITART, M. (2012a): «Towards a multimethodological approach to identification of funds of identity, small stories and master narratives», Narrative Inquiry, 22, pp. 173-180.

ESTEBAN-GUITART, M. (2012b): «La multi-metodología autobiográfica extendida (MAE). Una estrategia cualitativa para estudiar la identidad, los fondos de conocimiento y las formas de vida», REMA. Revista Electrónica de Metodología Aplicada, 17, pp. 51-64.

ESTEBAN-GUITART, M. y VILA, I. (2010): «Modelos culturales y retratos de identidad. Un estudio empírico con jóvenes de distintos contextos sociodemográficos», Estudios de Psicología, 31, pp. 173-185.

ESTEBAN-GUITART, M. y VILA, I. (2013): Experiencias en educación inclusiva. Vinculación escuela, familia y comunidad, Barcelona, Horsori.

ESTEBAN-GUITART, M., OLLER, J. y VILA, I. (2012): «Vinculando escuela, familia y comunidad a través de los fondos de conocimiento e identidad. Un estudio de caso con una familia de origen marroquí», Revista de Investigación en Educación, 10, pp. 21-34.

ESTEBAN-GUITART, M., NADAL, J. M. y VILA, I. (2008): «La construcción narrativa de la identidad a través del conflicto y la ventrilocuación», Glossa. An Ambilingual Interdisciplinary Journal, 4, pp. 130-145.

ESTEBAN-GUITART, M., NADAL, J. M., VILA, I. y ROSTAN, C. (2008): «Aspectos ambientales implicados en la construcción de la identidad en una muestra de adolescentes de la Universidad Intercultural de Chiapas», Medio Ambiente y Comportamiento Humano. Revista Internacional de Psicología Ambiental, 9, pp. 91-117.

FERREIRA, H, y PASUT, M. (1998): Técnicas grupales. Elementos para el aula flexible, Novedades educativas-Colección Recursos Didácticos, Argentina.

GEILFUS, F. (1997): 80 herramientas para el desarrollo participativo: Diagnóstico, planificación, monitoreo, evaluación, México, SAGARPA-IICA/México-INCA RURAL.

EMPIRIA. Revista de Metodología de Ciencias Sociales. N. ${ }^{\circ}$ 26, julio-diciembre, 2013, pp. 175-200. ISSN: 1139-5737, DOI: 10.5944/empiria.26.7157 
GIDDENS, A. (1997): Modernidad e identidad del yo: el yo y la sociedad en la época contemporánea, Barcelona, Península.

GIFRE, M., MONREAL, P. y ESTEBAN-GUITART, M. (2011): «El desarrollo de la identidad a lo largo del ciclo vital. Un estudio cualitativo y transversal», Estudios de Psicología, 32, pp. 227-241.

GIMÉNEZ, G. (2000): «Materiales para una teoría de las identidades sociales», en: VALENZUELA A., J.M. (Coord.) (2000): Decadencia y auge de las identidades: cultura nacional, identidad cultural y modernización, $2^{\mathrm{a}}$ Edición, pp. 45-78, México, El Colegio de la Frontera Norte.

GONZÁLEZ, N., MOLL, L. y AMANTI, C. (2005): Funds of knowledge. Theorizing practices in households, communities, and classrooms, Mahwah, Lawrence Erlbaum Associates.

GONZÁLEZ-PATIÑO, J. (2011): «Rutinas de la infancia urbana mediadas por la tecnologia: Un análisis visual», Papeles de Trabajo sobre Cultura, Educación y Desarrollo Humano, 7, pp. 1-16.

GONZÁLEZ REY, F. (1998): «Epistemología cualitativa y subjetividad», Revista Interamericana de Psicología, 32, pp. 139-167.

HALL, S. (1996): «Introducción: ¿Quién necesita 'identidad'?», en: HALL, S. y DU GAY, P., (comps.): Cuestiones de identidad cultural, pp. 13-39, Buenos Aires, Amorrortu.

HALL, S. (1999): «Identidad cultural y diáspora», en: CASTRO G., S, et.al., (comps.): Pensar (en) los intersticios: Teoría y práctica de la crítica poscolonial, pp. 131-145, Bogotá, Ceja.

HARTMAN, A. (1979): «Diagrammatic assessment of family relationships», Families in Society, 76, pp. 111-122.

HARTLEY, W. S. (1970): Manual for the twenty statements problem, Kansas City, Greater Kansas City Mental Health Foundation.

KÄES, R. (1976): El aparato psíquico grupal. Construcciones de grupo, Barcelona, Gedisa.

MAALOUF, A. (1999): Identidades asesinas, Madrid, Alianza.

MCADAMS, D. P. (2003): «Identity and the life story», en Autobiographical memory and the construcction of a narrative self. Development and cultural perspectives, Mahwah, Lawrence Erlbaum Associates, pp. 187-208.

MEAD, G. H. (1934): Mind, self and society, Chicago, University of Chicago Press.

MOLL, L. (1997): «Vygotski, la educación y la cultura en acción», en Hacía un currículum cultural. La vigencia de Vygotski en la educación, Madrid, Fundación Infancia y Aprendizaje, pp. 39-53.

MONTAÑÉS, S. M. (2009): Metodología y técnica participativa. Teoría y práctica de una estrategia de investigación participativa, Barcelona, UOC.

PAZ R., C. (1996): «Grupo y psicodrama», Anuario de Investigación del Departamento de Educación y Comunicación, México, UAM-X, pp. 707-712.

PEÑA-CUANDA, M. (2008): «Pensar la interpretación: la construcción del sentido en ciencias sociales», Liminar. Estudios Sociales y Humanísticos, 6, pp. 177-187.

PEÑA-CUANDA, M. (2010): «Diásporas, identidaes y movimientos pobalcionales no transnacionales», Liminar. Estudios Sociales y Humanísticos, 8, pp. 122-136.

POVEDA, D., CASLA, M., MESSINA, C., MORGADE, M., RUJAS, I., PULIDO, L. y CUEVAS, I. (2007): «The after school routines of literatura-devoted urban children», Children $\mathbf{\nabla}$ Geographies, 5, pp. 423-441.

EMPIRIA. Revista de Metodología de Ciencias Sociales. N. ${ }^{\circ}$ 26, julio-diciembre, 2013, pp. 175-200. ISSN: 1139-5737, DOI: 10.5944/empiria.26.7157 
RATNER, C. (2012): Macro cultural psychology: A political philosophy of mind, New York, Oxford University Press.

RIQUER F. (1992): «Identidad Femenina en la frontera entre la conciencia y la interacción social», en: TARRÉS, M.L., La voluntad de ser. Mujeres en los Noventa, pp. 51-64, México, El Colegio de México.

SAUBICH, X. y ESTEBAN, M. (2011): «Bringing funds of family knowledge to schoool. The living Morocco project», REMIE. Multidisciplinary Journal of Educational Research, 1, pp. 57-81.

TAJFEL, H. (1984): Grupos humanos y categorías sociales, Barcelona, Herder.

VALENZUELA A., J. M. (2000): «Identidades Culturales: Comunidades Imaginarias y contingentes», en: VALENZUELA A., J. M. (Coord.) (2000): Decadencia y auge de las identidades: cultura nacional, identidad cultural y modernización, $2^{\mathrm{a}}$ Edición, pp. 97-120, México, El Colegio de la Frontera Norte. 